\title{
Sekundärpräventive Antiplättchentherapie in der kardiologischen Rehabilitation
}

\author{
Maria M. Wanitschek · Hannes F. Alber
}

Eingegangen: 14. Oktober 2016 / Angenommen: 15. Dezember 2016 / Online publiziert: 13. Januar 2017 (C) Der/die Autor(en) 2018. Dieser Artikel ist eine Open-Access-Publikation.

Zusammenfassung Das über Jahre langfristig erhöhte kardiovaskuläre Risiko von Patienten/innen mit einem akuten Koronarsyndrom (ACS) ist eine Herausforderung. Rezente Resultate für eine über ein Jahr hinaus prolongierte duale Antiplättchentherapie haben einen ischämische Nutzen zum Preis einer erhöhten Blutungsgefahr aufgezeigt. Daher ist eine individuelle, sorgfältige und wiederholte Risiko-NutzenAbwägung essentieller Bestandteil eines optimierten Patientenmanagements, in dem Rehabilitationsärzte/ innen eine zentrale Rolle spielen können.

Schlüsselwörter Langzeit; verlängerte - duale Antiplättchentherapie · Sekundärprävention · akutes Koronarsyndrom $\cdot$ Myokardinfarkt $\cdot$ Rehabilitation

\section{Secondary prevention with antiplatelet therapy in cardiac rehabilitation}

Summary The long-term increased cardiovascular risk of patients with an acute coronary syndrome (ACS) is a challenging and common clinical problem. Recent evidence demonstrated an ischemic benefit for a prolonged dual antiplatelet therapy beyond the initial 12 months at the cost of an increased bleeding risk. Individual, careful and repeated risk-

\section{M. Wanitschek, M.D. · H. F. Alber, M.D.}

Universitätsklinik für Innere Medizin III, Medizinische

Universität Innsbruck, Anichstraße 35, 6020 Innsbruck, Österreich

\section{H. F. Alber, M.D. ( $₫)$}

Department Kardiologie, Reha-Zentrum Münster,

Gröben 700, 6232 Münster, Österreich

hannes.alber@i-med.ac.at

\section{H. F. Alber, M.D.}

Karl Landsteiner Institut für interdisziplinäre Forschung, Reha Zentrum Münster in Tirol, 6232 Münster, Österreich benefit analyses are essential for an optimized patient management in which cardiovascular rehabilitation providers may play a central role.

Keywords long-term; prolonged - dual antiplatelet therapy · secondary prevention · acute coronary syndrome $\cdot$ myocardial infarction $\cdot$ rehabilitation program

\section{Einleitung}

Die Therapie mit niedrig dosiertem Aspirin in Kombination mit einer zweiten Antiplättchensubstanz gehört seit mittlerweile Jahrzehnten zum Standard nach einem akuten Koronarsyndrom (ACS) bzw. nach Implantation von Stents oder bioresorbierbarer Gefäßstützen (BRS). Grundsätzlich zielt eine duale Antiplättchentherapie (DAPT) auf die Prävention von Stent-bezogenen und Atherosklerose-bedingten Ereignissen $a b$ [1]. War es vor wenigen Jahren noch häufig üblich sowohl nach einem ACS als auch nach Implantation eines medikamentenbeschichteten Stents (DES) eine DAPT prinzipiell für bis zu 12 Monate $\mathrm{zu}$ verordnen, so haben rezente Entwicklungen im Bereich der Stents, aber auch neue Erkenntnisse über die Ereignisrate spät nach einem ACS diese etwas vereinfachte, jedoch einheitliche Therapiedauer verändert. Bedingt durch technische Verbesserungen der DES (dünnere Stentstreben, geringere Medikamentendosis, Änderungen im Bereich der Polymere bzw. Polymer-freie Varianten) ist - rein aus Stent-Sicht betrachtet - eine Verkürzung der Dauer einer DAPT durchaus möglich. Eine Metaanalyse von 7 Studien (ITALIC, SECURITY, PRODIGY, RESET, OPTIMIZE, ISAR-SAFE, EXCELLENT), in denen Zweitgenerations-DES verwendet wurden, hat sich mit einer verkürzten ( $\leq 6$-monatigen) DAPT-Dauer im Vergleich $\mathrm{zu}$ einer 12-Monats-Strategie beschäftigt [2]. Ziada et al. haben hinsichtlich der Gesamtsterblichkeit 


\begin{tabular}{|c|c|c|}
\hline sKHK: & $\begin{array}{l}\text { nach BMS: } \\
\text { nach DES: } \\
\text { nach BVS: }\end{array}$ & $\begin{array}{l}\text { zumind. } 1 \text { Monat } \\
6 \text { Monate }^{1} \\
12 \text { Monate }^{2}\end{array}$ \\
\hline ACS: & grundsätzlich: & 12 Monate (unabhängig vom Stenttyp) ${ }^{3}$ \\
\hline ACS + nvVHF: & $\begin{array}{r}\text { niedriges/mittle } \\
\mathrm{Dr} \\
\mathrm{Do} \\
\mathrm{OA} \\
\text { hohes Blutungs } \\
\mathrm{Dr} \\
\mathrm{Do} \\
\mathrm{OA}\end{array}$ & $\begin{array}{l}\text { Angsrisiko (HAS-BLED 0-2) } \\
\text { herapie (OAC+C+ASS) über } 6 \text { Monate } \\
\text { herapie (OAC+C oder ASS) von Monat } 7 \text { bis } 12^{4} \\
\text { therapie nach } 12 \text { Monaten } \\
\text { AS-BLED } \geq 3 \text { ) } \\
\text { herapie (OAC+C+ASS) über } 4 \text { Wochen }{ }^{6} \\
\text { herapie (OAC+C oder ASS) von Monat } 2 \text { bis } 12^{4} \\
\text { therapie nach } 12 \text { Monaten }\end{array}$ \\
\hline sKHK + nvVHF: & \multicolumn{2}{|c|}{$\begin{array}{l}\text { CHADS-VASc } \geq 2 \text { / HAS-BLED } \leq 2 \text { und CHADS-VASc } \geq 2 \text { / HAS-BLED } \geq 3 \text { : } \\
\text { wie bei ACS+nvVHF und hohem Blutungsrisiko }\end{array}$} \\
\hline \multicolumn{3}{|c|}{$\begin{array}{l}\text { ürzung bei hohem Blutungsrisiko oder eine Verlängerung bei hohem ischämischen Risiko/niedrigem Blutungsrisiko kann überlegt werden (Ilb-Empfehlung). } \\
\text { t explizit in den Revaskularisations-Richtlinien } 2014 \text { angeführt. }\end{array}$} \\
\hline \multicolumn{3}{|c|}{$\begin{array}{l}\text { kürzung nach DES bei hohem Blutungsrisiko auf 3-6 Monate oder eine Verlängerung über ein Jahr hinaus nach sorgfältigem Abwägen des Blutungs- und } \\
\text { chen Risikos kann überlegt werde (IIb-Empfehlung) }\end{array}$} \\
\hline \multicolumn{3}{|c|}{$\begin{array}{l}\text { Itternative zu Clopidogrel kann bei einer doppelten antithrombotischen Therapie überlegt werden (d.h. OAC+1 Antiplättchensubstanz). Eine dreifache } \\
\text { mbotische Therapie kann bei sehr hohem ischämischen Risiko für bis zu } 12 \text { Monate überlegt werden. }\end{array}$} \\
\hline \multicolumn{3}{|c|}{$\begin{array}{l}\text { pelte antithrombotische Therapie (d.h. OAC+1 Antiplättchensubstanz) über ein Jahr hinaus kann bei sehr hohem koronaren Risiko überlegt werden. Eine duale } \\
\text { chentherapieals Alternative zu einer dreifachen oder doppeltenantithrombotischen Therapie (d.h. OAC+C und/oder A) kann bei einem CHA2DS2-VASc-Score } \\
\text { lännern) oder } 2 \text { (Frauen) überlegt werden. }\end{array}$} \\
\hline
\end{tabular}

Abb. 1 Dauer der dualen Antiplättchentherapie nach Koronarintervention. (Auf Basis der Revaskularisations-Richtlinien2014 [3] und NSTE-ACS-Richtlinien 2015 [4] der ESC); sKHK stabile koronare Herzkrankheit = stable coronary artery disease, ACS akutes Koronarsyndrom = acute coronary syndrome, $n v V$ $H F$ nicht-valvuläres Vorhofflimmern = non-valvular atrial fibrillation, $O A C$ orale Antikoagulation $=$ oral anticoagulation, $C$ Clopidogrel, $A S S$ Azetylsalizylsäure = acetylic salicylic acid, $B M S$ bare metal stent, $D E S$ drug eluting stent, $B V S$ bioabsorbierbare Gefäßstütze = bioabsorbable vascular scaffold, CHADS-VASc-
Score Score zur Beurteilung des Thromboembolierisikos bei nicht valvulärem Vorhofflimmern = score for the estimation of thromboembolic risk in patients with non-valvular atrial fibrillation, HAS-BLED Score zur Beurteilung des Blutungsrisikos = score for the estimation of the bleeding risk, empfohlen von den ESC Richtlinien zur Behandlung von Patienten/innen mit nicht valvulärem Vorhofflimmern = as suggested by the guidelines for the management of atrial fibrillation of the European Society of Cardiology [5] (adaptiert nach [6])

Tab. 1 Dauer und vorzeitige Abbruchrate der (dualen) Antiplättchentherapie in wichtigen ACS-DAPT-Studien

\begin{tabular}{|c|c|c|c|c|}
\hline Studienakronym & Randomisierte Therapie & DauermEDIAN (Exposure MEDIAN) der Therapie & \multicolumn{2}{|c|}{ Studienmedikations-Abbruchrate (\%) } \\
\hline CURE & C/A vs. A & 9 Mo (3-12) & C/A: 21,1 & $A: 18,8$ \\
\hline TRITON & C/A vs. P/A & 14,5 Mo & C/A: 6,3 & $P / A: 7,2^{b}$ \\
\hline PLATO & C/A vs. T/A & $9,2 \mathrm{Mo}(\mathrm{IQR} \approx 6-12)^{\mathrm{a}}$ & C/A: 21,5 & T/A: 23,4 \\
\hline TRILOGY & C/A vs. P/A & 14,8 Mo (IQR 8,2-23,6) & C/A: 22 & P/A: 24 \\
\hline
\end{tabular}

$(0,93[0,73-1,17])$, der Rate an Myokardinfarkten $(1,13$ $[0,88-1,44])$ oder Stentthrombosen $(1,28[0,83-1,98])$ keinen signifikanten Nachteil für eine verkürzte DAPT berichtet, die Rate an schweren Blutungen wurde jedoch durch die Reduktion der Therapiedauer halbiert (OR 0,51 [0,32-0,79]). Angesichts dieser und ähnlicher Daten sind die Empfehlungen für die Dauer (und auch Intensität) der antithrombotischen Therapie nach Stentimplantationen und nach akutem Koronarsyndrom (mit oder ohne gleichzeitig bestehender Indikation für eine orale Antikoagulation) in den letz- ten Jahren komplexer geworden, um den Patienten/ innen eine möglichst individualisierte Therapie anbieten zu können. Abb. 1 liefert einen Überblick über die diesbezüglichen Empfehlungen der europäischen kardiologischen Gesellschaft in den Richtlinien zur myokardialen Revaskularisation aus dem Jahr 2014 [3] sowie zur Behandlung von Nicht-ST-HebungsAkute Koronarsyndrome (NSTE-ACS) von 2015 [4].

Angesichts der durch die technischen Entwicklungen im Stentsektor bedingten, potentiellen Reduktion der Dauer einer dualen Antiplättchentherapie stellt 
Abb. 2 DAPTEinnahme/ Beendigungsrate nach 30 Tagen, einem und zwei Jahren in der PARIS prospektiven, Observationsstudie-Mehran et al. [7]

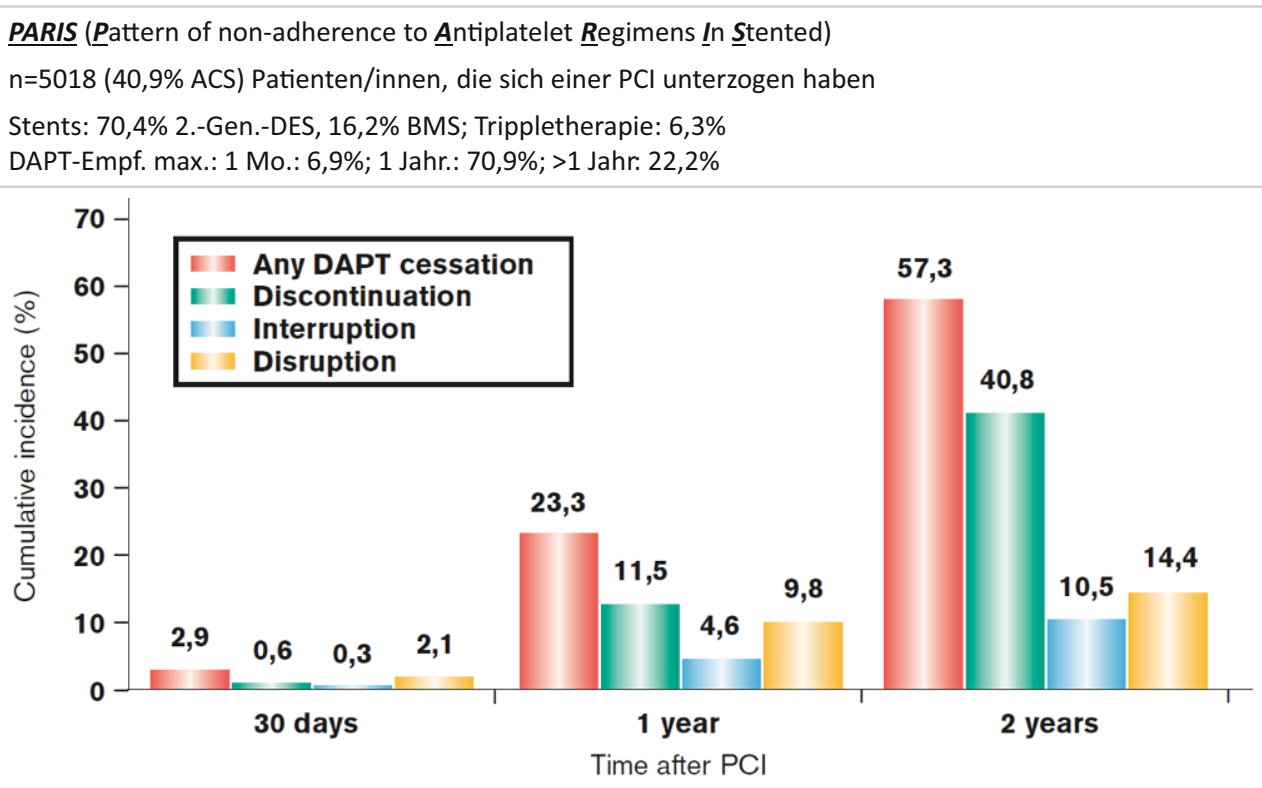

Discontinuation ist die auf ärztlichen Rat empfohlene Beendigung, Interruption bezieht sich auf eine (meist durch einen operativen Eingriff bedingte) Unterbrechung für bis zu 14 Tage. Disruption bezeichnet den Abbruch bedingt durch eine Blutung oder durch Non-Compliance sich - gerade auch in der Rehabilitationsmedizin - die Frage, welcher Patient/welche Patientin mit koronarer Herzkrankheit möglicherweise sogar von einer verlängerten DAPT profitieren könnte. Diesbezüglich fasst Tab. 1 die medianen Therapiedauern sowie die Studienmedikationsabbruchraten großer randomisierter Studien zu diesem Thema zusammen. Anhand der Interquartilenbereiche ist $\mathrm{zu}$ erkennen, dass in diesen Megatrials sowohl deutlich kürzere, als auch deutlich längere Therapiedauern als 12 Monate durchgeführt wurden. Auch in PARIS (Pattern of non-adherence to Antiplatelet Regimens In Stented patients), einer prospektiven Beobachtungsstudie an 5018 Patienten/innen, die sich einer perkutanen Koronarintervention (PCI) unterzogen haben $(40,9 \%$ hatten ein ACS), zeigte sich, dass 2 Jahre nach erfolgreich durchgeführter PCI 57,3\% der Patienten/innen eine DAPT beendet hatten [7]. Anders betrachtet nahmen fast $43 \%$ der Patienten/innen 2 Jahre nach einer PCI noch immer eine DAPT ein. Zudem wurde bereits initial 22,2\% der Patienten/innen eine DAPT für länger als 1 Jahr durch das behandelnde Interventionszentrum empfohlen (Abb. 2).

\section{Langfristig erhöhtes Risiko nach einem akuten Koronarsyndrom}

Entsprechend einer schwedischen Registeranalyse von Jernberg et al. ist die Ereignisrate bei Patienten/ innen nach einem akuten Myokardinfarkt innerhalb des ersten Jahres bei 18,3\% [8]. Dies gilt nur für jene Patienten/innen, die ihren Index-Myokardinfarkt bis zur Entlassung überlebt haben. Mehr als die Hälfte der in diesem ersten Jahr auftretenden Ereignisse sind letal, wobei $2 / 3$ der Todesfälle kardiovaskulär be- dingt sind. Somit besteht im ersten Jahr nach einem Myokardinfarkt ein substanziell hohes Ereignis- und Mortalitätsrisiko. Jene Patienten/innen ( $n=76.687$ ), die das erste Jahr nach Myokardinfarkt ohne Ereignis überleben, werden als stabile Post-MyokardinfarktPatienten/innen bezeichnet. Der Begriff „stabil“ kann jedoch durchaus als irreführend betrachtet werden, da diese Menschen in den nächsten 24 Monaten (also in den Jahren 2 und 3 nach dem Index-Myokardinfarkt) eine nach wie vor hohe $(17,7 \%$ ige) Wahrscheinlichkeit, ein erneutes kardiovaskuläres Ereignis zu erleiden, haben. Somit geht aus diesen Registerdaten klar hervor, dass sicherlich die höchste Ereignisrate im ersten Jahr nach Myokardinfarkt besteht, aber auch, dass in den darauf folgenden Jahren Patienten/innen unverändert in einem zweistelligen Prozentbereich kardiovaskulär gefährdet sind. Eine ähnlich hohe Ereignisrate in Post-Myokardinfarkt-Patienten/innen konnte auch in der „Osaca Acute Coronary Insufficiency" Studie mit einem Nachbeobachtungszeitraum von bis zu 5 Jahren bei 7870 Patienten/innen beobachtet werden [9]. Besonders gefährdet sind dabei jene Menschen mit mehr als einem Myokardinfarkt in ihrer Anamnese. Mit einem Follow-up von bis zu 20 Jahren konnten Nauta et al. in einer Arbeit aus dem Jahr 2012 ein besonders hohes Risiko für Patienten/ innen mit Diabetes mellitus und Myokardinfarkt in der Vorgeschichte aufzeigen [10]. Dementsprechend wurde in einer Analyse der Framingham Heart Study bereits zu Beginn dieses Jahrtausends eine dramatische Reduktion der Lebenserwartung der Patienten/ innen mit der Vorgeschichte eines akuten Myokardinfarkts im Vergleich zur Patienten ohne kardiovaskuläre Erkrankung berichtet. In Abhängigkeit des $\mathrm{Ge}$ schlechts und des Alters reduziert sich durch einen 
Myokardinfarkt die Lebenserwartung von 4,3 Jahren (bei 80-jährigen Männern) bis zu 14,9 Jahren (bei 50jährigen Frauen) [11]. Diese Fakten berücksichtigend ist somit jeder Arzt/jede Ärztin, der/die mit der Behandlung von Post-Myokardinfarkt-Patienten/innen betraut ist, gefordert, möglichst effektiv und langfristig Risiko-senkend zu agieren. Mehrere Studien und Metaanalysen der letzten Jahre haben rehabilitativen Maßnahmen einen Mortalitäts-Benefit bescheinigt $[12,13]$. Die Teilnahme an multidisziplinären Rehabilitationsprogrammen erhält daher nicht zu unrecht in diversen Richtlinien durchwegs eine vorrangige Empfehlung [14]. Neben der Steigerung der körperlichen Leistungsfähigkeit, der Patienten/innen-Aufklärung und -Erziehung sowie der psychologischen Betreuung bieten multiprofessionelle Rehabilitationsprogramme auch die Möglichkeit einer intensiven Beschäftigung mit der medikamentösen Risikominimierung von Patienten/innen mit koronarer Herzkrankheit, insbesondere von Post-Myokardinfarkt-Patienten/innen. In kurzer Zeit häufige und regelmäßige Arzt-Patienten-Kontakte erlauben eine detaillierte Kontrolle/ Überprüfung des Wirkungs- und vor allem auch Nebenwirkungsprofils verschiedener Substanzklassen, unter anderem auch von Antiplättchen-Medikamenten.

Das langfristig erhöhte kardiovaskuläre Risiko von Post-Myokardinfarkt-Patienten/innen einerseits und der Nutzen einer DAPT speziell im ersten Jahr nach einem ACS andererseits werfen die Frage auf, wie ausgeprägt das Risiko-Nutzen-Verhältnis für eine über 12 Monate verlängerte DAPT nach einem ACS im individuellen Fall sein kann bzw. ist. Diese Einzelfallentscheidung ist in den NSTE-ACS-ManagementRichtlinien der europäischen kardiologischen Gesellschaft 2015 [4] mit einer Klasse-IIb-Empfehlung auf einem Evidenzniveau A $[15,16]$ folgender Maßen verankert: „P2 $\mathrm{Y}_{12}$ inhibitor administration in addition to aspirin beyond one year may be considered after careful assessment of the ischaemic and bleeding risks of the patient.“

\section{Datenlage zu einer prolongierte DAPT}

Die Datenlage, auf der die Möglichkeit einer über ein Jahr prolongierten DAPT gegeben ist, besteht neben den beiden in der NSTE-ACS-Richtlinie zitierten Studien (DAPT und PEGASUS) auch aus Subanalysen anderer Arbeiten. Obwohl in der „Clopidogrel for High Atherothrombotic Risk and Ischemic Stabilization, Management and Avoidance (CHARISMA)" Studie zu einer verlängerten DAPT mit Clopidogrel und Aspirin über 28 Monate kein Vorteil für eine Prolongation in der Gesamtpopulation zu sehen war, ergab eine Subgruppenanalyse, dass jene 9478 Patienten/innen mit einer zum Randomisierungs-Zeitpunkt bestehenden atherosklerotischen Erkrankung sehr wohl eine signifikante 17,1 \%ige Risikoreduktion unter der DAPT im kombinierten ischämischen Endpunkt bestehend aus kardiovaskulärer Mortalität, Myokardinfarkt oder Schlaganfall hatten [17]. Erwartungsgemäß war auch in dieser Studie die Rate an moderaten bis schweren Blutungen signifikant erhöht, wobei der zeitliche Verlauf dieser Blutungen gezeigt hat, dass dieses Risiko vor allem in den ersten 12 Monaten mit 2,0 versus $1,1 \%$ erhöht, danach jedoch für beide Gruppen mit einer Blutungsrate von ca. 0,8\% pro Jahr stabil und vergleichbar war.

Eine intensivierte Plättchenhemmung nach einem akuten Koronarsyndrom wurde auch in der „Thrombin Receptor Antagonist Vorapaxar in Acute Coronary Syndrom" (TRACER) Studie mit dem neuen oralen Protease-Activated Receptor 1 (PAR-1) Antagonist Vorapaxar an 12.944 Patienten untersucht [18]. Diese Studie wurde nach ca. 500 Tagen vorzeitig beendet, da die Rate an moderaten und schweren Blutungen in der Vorapaxar-Gruppe um $35 \%$ und die Rate an intrakraniellen Blutungen um das 3,39-fache erhöht waren. Diese inakzeptabel hohe Blutungsrate führte zum Abbruch der Studie. Die Analyse des ischämischen Endpunktes ergab jedoch eine signifikante Reduktion von 16,4 auf 14,7\% für die Kombination aus kardiovaskulärer Mortalität, Myokardinfarkt oder Schlaganfall. Anhand der Post-hoc-Analyse der negativen CHARISMAStudie sowie der Analyse der vorzeitig beendeten TRACER-Studie wird das Dilemma für die tägliche Praxis offenkundig; es gilt die richtige Balance zwischen Blutungsgefährdung und ischämischen Nutzen im Einzelfall richtig einzuschätzen.

Neben der richtigen Patientenselektion spielt sicherlich auch die Intensität der Plättchenhemmung eine zentrale Rolle; speziell TRACER hat dies aufgezeigt. In der oben bereits erwähnten DAPT-Studie wurde eine Kombination aus Prasugrel und Clopidogrel nach Stent-Implantation untersucht. Dabei wurden 9961 Patienten innerhalb von $72 \mathrm{~h}$ nach StentImplantation eingeschlossen. Mehr als ein Drittel der Patienten $(37,7 \%)$ hatten als Indikation für die PCI eine stabile Angina pectoris, zwei Drittel erhielten Clopidogrel und ein Drittel Prasugrel. Die Randomisierung zur verlängerten DAPT erfolgte nach 12 Monaten für zusätzliche 18 Monate. Voraussetzung dafür war unter anderem, dass die Patienten kein Blutungsereignis im ersten Jahr unter der in der Routine verwendeten DAPT aufwiesen. Aufgrund dieser Einschlusskriterien ist klar, dass diese DAPT-Studie ein heterogenes „post-PCI-Patientenkollektiv“ eingeschlossen hat. Da nur 10,4\% STEMI-Patienten/Innen und lediglich 15,5\% NSTEMI-Patienten/Innen eingeschlossen wurden, ist die Aussage der Studie für das Hochrisikokollektiv der stabilisierten Post-Myokardinfarkt-Patienten/innen naturgemäß eingeschränkt. Sie hat jedoch aufgezeigt, dass die Verlängerung der Gabe von Aspirin und eines Thienopyridins über 12 Monate hinaus (bis zu 30 Monate) den kombinierten ischämischen Endpunkt aus Mortalität, Myokardinfarkt oder Schlaganfall um $29 \%$ von $5,9 \%$ auf $4,3 \%$ signifikant senken kann. Dies ging einher mit einer signifikanten 
Abb. 3 Eine sorgfältige Evaluierung des kardiovaskulären Risikos und des Risikos für Blutungen sollte zu jedem Zeitpunkt mit Hinblick auf die individuelle Empfehlung bezüglich der Dauer einer DAPT erfolgen. Unter anderem sollten folgende Faktoren berücksichtigt werden. (Adaptiert nach Weiss et al. [20])

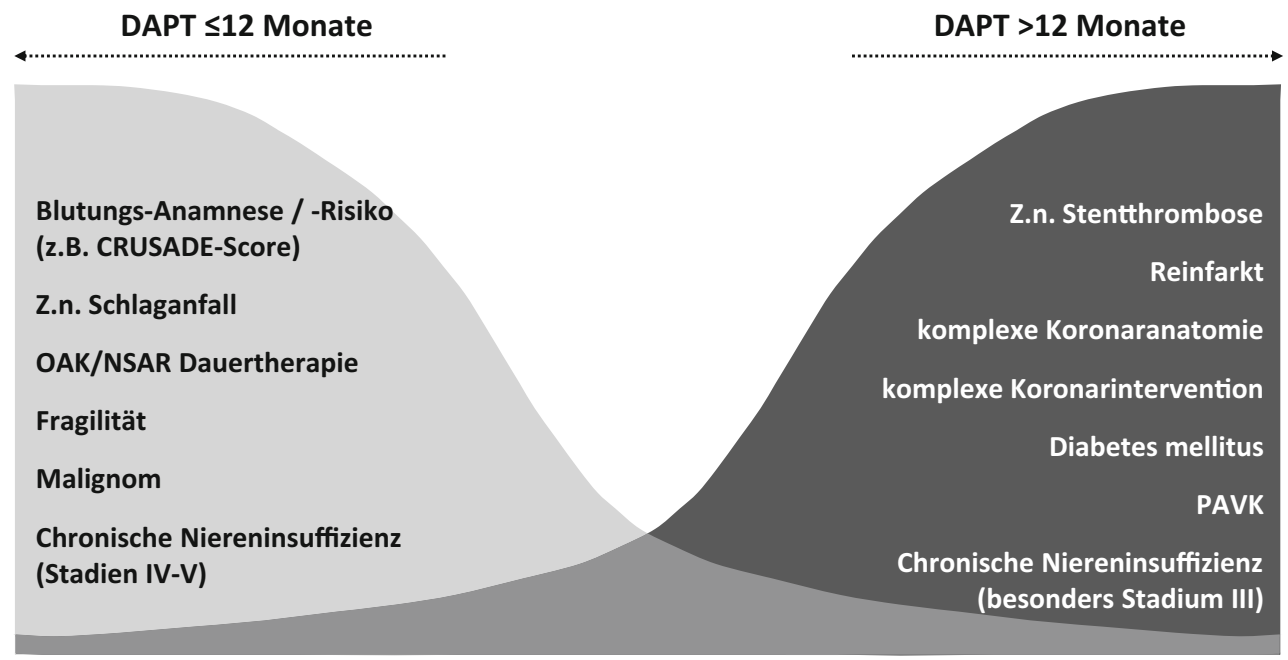

Zunahme der moderaten sowie BARC [19] Typ 2 und Typ 3 Blutungen. Fatale Blutungen waren mit 0,1\% selten und nicht statistisch signifikant unterschiedlich. Die Analyse der nicht kardiovaskulären Mortalität ergab einen statistisch signifikanten Nachteil für die prolongierte DAPT (48 versus 22 Todesfälle, wobei blutungsbezogene Todesfälle mit 11 versus 3 deutlich weniger auffällig waren als der Unterschied in den Tumor-bezogenen Todesfällen mit 31 versus 14). Somit kann dieses Phänomen nicht direkt der Verlängerung der intensivierten Antiplättchentherapie zugeschrieben werden. In Einklang mit den Subgruppenanalysen von CHARISMA und die Auswertung von TRACER hat jedoch auch DAPT gezeigt, wie wichtig das individuelle Abwägen von Blutungs- und Ischämie-Risiko ist. Eine ebenso interessante und für die tägliche Praxis relevante Erkenntnis der DAPT-Studie ist, dass in den ersten drei Monaten nach Absetzen einer dualen Antiplättchentherapie bei einem gemischten Post-PCIPatientenkollektiv die Ereignisrate am höchsten ist. Dieser Fakt hängt wohl nicht mit einem ReboundPhänomen des Absetzens der Antiplättchen-Behandlung, sondern am ehesten mit einem Wegfall des ischämischen Schutzes einer solchen zusammen. In der klinischen Routine sowohl eines Akut- als auch eines Rehabilitationskrankenhauses sollte daher die Entscheidung für oder gegen eine prolongierte DAPT noch während der laufenden Kombinationsbehandlung des ersten Jahres getroffen werden. Die größte und spezifische Studie zu einer prolongierten DAPT in Hochrisiko-Patienten/innen in der stabilisierten PostMyokardinfarkt-Phase wurde letztes Jahr publiziert. Die „Prevention of Cardiovascular Events in Patients with prior Heart Attack using Ticagrelor compared to Placebo on an Background of Aspirin - thrombolysis in myocardial infarction 54 - PEGASUS-TIMI54" Studie hat zu diesem Zweck ein bis drei Jahre nach einem akuten Koronarsyndrom 21.162 Patienten/innen zu Placebo oder Ticagrelor in zwei verschiedenen Dosierungen $(2 \times 60$ und $2 \times 90 \mathrm{mg}$ tgl. $)$ randomisiert. Neben der Vorgeschichte eines Myokardinfarktes mussten die Patienten/innen zumindest einen zusätzlichen Risikofaktor wie Diabetes mellitus, Reinfarkt, Alter $\geq 65$ Jahre, dokumentierte Mehrgefäßerkrankung oder chronische Niereninsuffizienz aufweisen.

Da die Resultate für $2 \times 90 \mathrm{mg}$ vergleichbar mit jenen von $2 \times 60 \mathrm{mg}$ waren und die Zulassung der europäischen Arzneimittelbehörde nur für die $60 \mathrm{mg}$ Dosierung im Frühjahr 2016 erfolgte, werden nachfolgend nur Resultate der Dosierung von $2 \times 60 \mathrm{mg}$ Ticagrelor als prolongierte DAPT in der stabilen PostMyokardinfarkt-Phase weiter erläutert. Die Ereignisrate für den Endpunkt kardiovaskuläre Mortalität, Myokardinfarkt oder Schlaganfall über 3 Jahre war in diesem Hochrisiko-Patientenkollektiv 9,04\% unter Aspirin und Placebo. Daran kann erkannt werden, dass durchaus eine gewisse Selektion vorhanden war, da diese Ereignisrate deutlich niedriger im Vergleich $\mathrm{zu}$ Registeranalysen ist (siehe oben die Arbeit von Jernberg). Nichtsdestotrotz konnte durch die Verlängerung der DAPT mit Ticagrelor $(2 \times 60 \mathrm{mg})$ die Ereignisrate auf $7,77 \%$ um $16 \%$ signifikant reduziert werden. Der Benefit in den einzelnen Komponenten des kombinierten Endpunkts war für die Myokardinfarkt- und Schlaganfall-Rate mit jeweils einem $p$-Wert von 0,03 signifikant. Die kardiovaskuläre Sterblichkeit wurde um relative $17 \%$ statistisch formal nicht signifikant $(p$-Wert 0,07$)$ gesenkt. Somit hat sich in dieser für Patienten/innen in der stabilisierten PostMyokardinfarkt-Phase spezifischen Studie ebenfalls der Benefit einer verlängerten DAPT herauskristallisiert. Gleichzeitig mit einem ischämischen Nutzen stieg auch die Rate an geringen und schweren Blutungen nach der TIMI-Klassifikation signifikant an. Tödliche oder intrakranielle Blutungen waren jedoch nicht unterschiedlich. Aus zusätzlichen Analysen dieser PEGASUS-Studie lässt sich auch in einem als „irreversiblen Schaden“ definierten Endpunkt, der ischämische Endpunkte sowie intrakranielle und fatale Blutungsereignisse zusammenfasst, ein positives Risiko-Nutzen-Verhältnis ableiten. Der Vorteil gilt primär für Patienten/innen, bei denen vor Rando- 
misierung zur prolongierten DAPT die Phase einer Aspirin-Monotherapie weniger als 30 Tage gedauert hat. Dies zeigt wieder auf, dass die Entscheidung zu einer prolongierten DAPT schon während der ersten 12 Monate gefällt und das Risiko für Blutungen in dieser Phase laufend evaluiert werden muss. Eines von vielen Entscheidungskriterien, die in Abb. 3 - naturgemäß unvollständig - aufgelistet sind und ausführlich in einem Konsensusmanuskript diskutiert worden sind [20], stellt die chronische Niereninsuffizienz im Stadium III dar. Für Patienten/innen mit dieser Komorbidität scheint eine besondere Effektivität gegeben zu sein [21].

\section{Bedeutung einer prolongierten DAPT nach ACS für die Rehabilitationsmedizin und Zusammen- fassung}

Oben angeführte Daten zeigen einerseits das über Jahre persistierende hohe kardiovaskuläre Risiko von Patienten/innen mit Anamnese eines akuten Koronarsyndroms auf und veranschaulichen andererseits die zentrale Bedeutung einer über längere Zeit geführten DAPT unter sorgfältiger, individueller und wiederholter Risiko-Nutzen-Abwägung. Der richtige Zeitpunkt für oder gegen die Entscheidung zum Weiterführen einer solchen ist sicherlich noch während der initialen 12 Monate. Die Ein- und Ausschlusskriterien der wegweisenden PEGASUS-Studie sollten berücksichtigt werden, jedoch kann aus weiterführenden Analysen von Langzeit-DAPT-Studien abgeleitet werden, dass beispielsweise auch die Komplexität einer Koronarintervention Berücksichtigung finden sollte. In diesem Zusammenhang wurde rezent in einem gepoolten Datensatz bei beinahe 10.000 Patienten/ innen ein Vorteil für eine Langzeit-DAPT primär in jener Gruppe mit komplexer PCI festgestellt [22]. Somit ist im Überlegungs- und Entscheidungsprozess bereits das Interventionszentrum gefordert, entsprechende Anmerkungen systematisch zu machen. Auch können zu diesem frühen Zeitpunkt im Management eines ACS klare Risikofaktoren für Blutungen (z. B. Zustand nach intrazerebraler Blutung, laufende Dialysetherapie, Indikation $\mathrm{zu}$ einer oralen Antikoagulation ...) festgestellt werden. Im weiteren Verlauf bietet jeder Arzt-Patienten-Kontakt die Möglichkeit, eventuell neu aufgetretene Nebenwirkungen der in diesem ersten Jahr laufenden DAPT bzw. neu vorhandene Kontraindikationen wiederholt zu evaluieren. In dieser Phase findet im Regelfall auch die Teilnahme an einem Rehabilitationsprogramm statt. Damit ist klar, welche Rolle die Rehabilitationsmedizin bei der Entscheidung für oder gegen einer über 12 Monate hinaus prolongierte DAPT trägt. Da - sofern die Einund Ausschlusskriterien der oben zitierten Studien für den/die individuelle/n Patienten/in abgeklärt sind eine prolongierte DAPT potenziell für jede/n ACSPatienten/in grundsätzlich eine Option darstellen kann, ist die akribische Suche nach sowie das genaue
Überprüfen und Korrigieren von potenziellen Risikofaktoren für Blutungen in den ersten 12 Monaten nach ACS von vorrangiger Bedeutung. Eine diesbezüglich klare Empfehlung bei der Entlassung aus einem ambulanten oder stationären Rehabilitationsprogramm würde zur individuellen Optimierung der DAPT nach ACS beitragen.

Open access funding provided by University of Innsbruck and Medical University of Innsbruck.

Interessenkonflikt M.M. Wanitschek und H.F. Alber geben an, dass kein Interessenkonflikt besteht.

Open Access Dieser Artikel wird unter der Creative Commons Namensnennung 4.0 International Lizenz (http:// creativecommons.org/licenses/by/4.0/deed.de) veröffentlicht, welche die Nutzung, Vervielfältigung, Bearbeitung, Verbreitung und Wiedergabe in jeglichem Medium und Format erlaubt, sofern Sie den/die ursprünglichen Autor(en) und die Quelle ordnungsgemäß nennen, einen Link zur Creative Commons Lizenz beifügen und angeben, ob Änderungen vorgenommen wurden.

\section{Literatur}

1. Bhatt DL, Fox KA, Hacke W, Berger PB, Black HR, Boden WE, Cacoub P, Cohen EA, Creager MA, Easton JD, Flather MD, Haffner SM, Hamm CW, Hankey GJ, Johnston SC, Mak KH, Mas JL, Montalescot G, Pearson TA, Steg PG, Steinhubl SR, Weber MA, Brennan DM, Fabry-Ribaudo L, Booth J, Topol EJ, CHARISMA Investigators. Clopidogrel and aspirin versus aspirin alone for the prevention of atherothrombotic events. NEngl J Med.2006;20;354(16):1706-17,Apr.

2. Ziada KM, Abdel-Latif A, Charnigo R, Moliterno DJ. Safety of an abbreviated duration of dual antiplatelet therapy ( $\leq 6$ months) following second-generation drug-eluting stents for coronary artery disease: A systematic review and meta-analysis of randomized trials. Catheter Cardiovasc Interv. 2016;87(4):722-32, Mar.

3. Windecker S, Kolh P, Alfonso F, Collet JP, Cremer J, Falk V, Filippatos G, Hamm C, Head SJ, Jüni P, Kappetein AP, Kastrati A, Knuuti J, Landmesser U, Laufer G, Neumann FJ, Richter DJ, Schauerte P, Sousa Uva M, Stefanini GG, Taggart DP, Torracca L, Valgimigli M, Wijns W, Witkowski A. 2014 ESC/EACTS Guidelines on myocardial revascularization: The Task Force on Myocardial Revascularization of the European Society of Cardiology (ESC) and the European Association for Cardio-Thoracic Surgery (EACTS)Developed with the special contribution of the European Association of Percutaneous Cardiovascular Interventions (EAPCI). Eur Heart J.2014 Oct 1;35(37):2541-619.

4. Roffi M, Patrono C, Collet JP, Mueller C, Valgimigli M, Andreotti F, et al. ESC Guidelines for the management of acute coronary syndromes in patients presenting without persistent ST-segment elevation: Task Force for the Management of Acute Coronary Syndromes in Patients Presenting without Persistent ST-Segment Elevation of the European Society of Cardiology (ESC). Eur Heart J. 2016;37(3):267-315. doi: 10.1093/eurheartj/ehv320. Epub 2015Aug 29 .

5. Kirchhof P, Benussi S, Kotecha D, Ahlsson A, Atar D, Casadei B, Castella M, Diener HC, Heidbuchel H, Hendriks J, Hindricks G, Manolis AS, Oldgren J, Popescu BA, Schotten U, Van Putte B, Vardas P, Agewall S, Camm J, Baron Esquivias G, Budts W, Carerj S, Casselman F, Coca A, De Caterina R, 
Deftereos S, Dobrev D, Ferro JM, Filippatos G, Fitzsimons D, Gorenek B, Guenoun M, Hohnloser SH, Kolh P, Lip GY, Manolis A, McMurray J, Ponikowski P, Rosenhek R, Ruschitzka F, Savelieva I, Sharma S, Suwalski P, Tamargo JL, Taylor CJ, Van Gelder IC, Voors AA, Windecker S, Zamorano JL, Zeppenfeld K. 2016 ESC Guidelines for the management of atrial fibrillation developed in collaboration with EACTS. Eur Heart J.2016 Oct 7;37(38):2893-962.

6. Alber H. Journal für Kardiologie - Austrian Journal of Cardiology 2016;23(9-10):242.

7. Mehran R, Baber U, Steg PG, Ariti C, Weisz G, Witzenbichler B, Henry TD, Kini AS, Stuckey T, Cohen DJ, Berger PB, Iakovou I, Dangas G, Waksman R, Antoniucci D, Sartori S, Krucoff MW, Hermiller JB, Shawl F, Gibson CM, Chieffo A, Alu M, Moliterno DJ, Colombo A, Pocock S. Cessation of dual antiplatelet treatment and cardiac events after percutaneous coronary intervention (PARIS): 2 year results from a prospective observational study. Lancet. 2013;23;382(9906):1714-22, Nov.

8. JernbergT, HasvoldP,Henriksson M,HjelmH, Thuresson M, JanzonM.Cardiovascularriskin post-myocardialinfarction patients: nationwide real world data demonstrate the importance of a long-term perspective. Eur Heart J. 2015;14;36(19):1163-70, May.

9. Nakatani D, Sakata Y, Suna S, Usami M, Matsumoto S, Shimizu M, Sumitsuji S, Kawano S, Ueda Y, Hamasaki T, Sato H, Nanto S, Hori M, Komuro I. Osaka Acute Coronary Insufficiency Study (OACIS) Investigators. Incidence, predictors, and subsequent mortality risk of recurrent myocardial infarction in patients following discharge for acute myocardial infarction. Circ J.2013;77(2):439-46.

10. Nauta ST, Deckers JW, Akkerhuis KM, van Domburg RT. Short- and long-term mortality after myocardial infarction in patients with and without diabetes: changes from 1985 to 2008. Diabetes Care. 2012;35(10):2043-7, Oct.

11. Peeters A, Mamun AA, Willekens F, Bonneux L. A cardiovascular life history. A life course analysis of the original Framingham Heart Study cohort. Eur Heart J. 2002;23(6):458-66, Mar.

12. Anderson L, Oldridge N, Thompson DR, Zwisler AD, Rees K, Martin N, Taylor RS. Exercise-Based Cardiac Rehabilitation for Coronary Heart Disease: Cochrane Systematic Review and Meta-Analysis. J Am Coll Cardiol. 2016;5;67(1):1-12, Jan.

13. de Vries H, Kemps HM, van Engen-Verheul MM, Kraaijenhagen RA, Peek N. Cardiac rehabilitation and survival in a large representative community cohort of Dutch patients. Eur Heart J. 2015;21;36(24):1519-28, Jun.

14. Sandesara PB, LambertCT, Gordon NF, Fletcher GF, Franklin BA, Wenger NK, Sperling L. Cardiac rehabilitation and risk reduction: time to "rebrand and reinvigorate". J Am Coll Cardiol. 2015;3;65(4):389-95, Feb.

15. Mauri L, Kereiakes DJ, Yeh RW, Driscoll-Shempp P, Cutlip DE, Steg PG, Normand SL, Braunwald E, Wiviott SD, Cohen DJ, Holmes DR Jr, Krucoff MW, Hermiller J, Dauerman HL, Simon DI, Kandzari DE, Garratt KN, Lee DP, Pow TK, Ver LP, Rinaldi MJ, Massaro JM. Twelve or 30 months of dual antiplatelet therapy after drug-eluting stents. N Engl J Med. 2014;371:2155-66.

16. Bonaca MP, Bhatt DL, Cohen M, Steg PG, Storey RF, Jensen EC, Magnani G, Bansilal S, Fish MP, Im K, Bengtsson O, Oude Ophuis T, Budaj A, Theroux P, Ruda M, Hamm C, Goto S, Spinar J, Nicolau JC, Kiss RG, Murphy SA, Wiviott SD, Held P, Braunwald E, Sabatine MS. Long-term use of ticagrelor in patients with prior myocardial infarction. N Engl J Med. 2015;372:1791-800.

17. Bhatt DL, Flather MD, Hacke W, Berger PB, Black HR, Boden WE, Cacoub P, Cohen EA, Creager MA, Easton JD, Hamm CW, Hankey GJ, Johnston SC, Mak KH, Mas JL, Montalescot G, Pearson TA, Steg PG, Steinhubl SR, Weber MA, Fabry-Ribaudo L, Hu T, Topol EJ, Fox KA, CHARISMA Investigators. Patients with prior myocardial infarction, stroke, or symptomatic peripheral arterial disease in the CHARISMA trial. J Am Coll Cardiol. 2007;15;49(19):1982-8, May.

18. Tricoci P, HuangZ, Held C, Moliterno DJ, Armstrong PW, Van de Werf F, et al. TRACER Investigators. Thrombin-receptor antagonist vorapaxar in acute coronary syndromes. N Engl JMed.2012;366(1):20-33.

19. Mehran R, Rao SV, Bhatt DL, Gibson CM, Caixeta A, Eikelboom J, Kaul S, WiviottSD, Menon V, Nikolsky E, Serebruany V, Valgimigli M, Vranckx P, Taggart D, Sabik JF, Cutlip DE, Krucoff MW, Ohman EM, Steg PG, White H. Standardized bleeding definitions for cardiovascular clinical trials: a consensus report from the Bleeding Academic Research Consortium. Circulation. 2011;123(23):2736-47.

20. Weiss TW, Aichinger J, Huber K, Speidl WS, Watzinger N, et al. Expert position paper on prolonged dual antiplatelet therapy in secondary prevention following myocardial infarction. Wien Klin Wochenschr. 2016;128(11-12):450-7. doi: 10.1007/s00508-016-1016-7. Epub2016Jun 9.

21. Magnani G, Storey RF, Steg G, Bhatt DL, Cohen M, et al. Efficacy and safety of ticagrelor for long-term secondary prevention of atherothrombotic events in relation to renal function: insights from the PEGASUS-TIMI 54 trial. Eur Heart J. 2016;37(4):400-8. doi: 10.1093/eurheartj/ehv482. Epub2015Oct5.

22. Giustino G, Chieffo A, Palmerini T, Valgimigli M, Feres F, Abizaid A, et al. Efficacy and Safety of Dual Antiplatelet Therapy After Complex PCI.J Am Coll Cardiol. doi: 10.1016/ j.jacc.2016.07.760. [Epub ahead of print] 